\title{
IMPACT OF CAROTID ENDARTERECTOMY ON COGNITIVE PERFORMANCE AND DEPRESSIVE SYMPTOMS
}

\author{
Elina Pucite ${ }^{1}$, Marius Slisers ${ }^{2}$, Evija Miglane ${ }^{3}$, Dainis Krievins ${ }^{4}$, \\ Renars Erts $^{5}$, Kristaps Jurjans ${ }^{6}$, Ildze Krievina ${ }^{7}$
}

\begin{abstract}
OBJECTIVES: This study aims to evaluate the impact of carotid endarterectomy (CEA) on cognitive performance in patients with severe carotid disease and depressive symptoms, and to explore the possible associations between certain demographics, clinical characteristics, and cognitive function and depression.

MATERIALS AND METHODS: The study included 48 patients, who were referred for endarterectomy. Cognitive function was assessed using the Montreal Cognitive Assessment (MoCA) scale, while depressive symptoms were assessed using the patient health questionnaire(PHQ-9) scale. An assessment of cognitive and depressive symptoms was performed 1-3 days before surgery, and then six months after.

RESULTS: A paired sample t-test found that the difference in the mean MoCA score between the before $(\overline{\mathrm{X}}=23.37 ; \mathrm{SD} \pm 3.27)$ and the after $(\overline{\mathrm{X}}=24.69 ; \mathrm{SD} \pm 3.68)$ surgery results was $1.32(95 \% \mathrm{CI}=0.48-2.16 ; p=$ 0.003 ; Cohen's $d$ value $=0.95$ ). A paired sample t-test showed that a decrease in mean PHQ-9 score of $>10$ for patients six months after CEA $(7.5 \pm 4.6)$ was statistically significant $(p=0.019$; Cohen's $d$ value $=1.32)$ compared with the PHQ-9 scores at baseline (12.6 \pm 2.8$)$

CONCLUSION: Carotid artery endarterectomy seems to have beneficial effects on the course of cognitive impairment and depressive symptoms in patients with severe carotid artery stenosis. Demographic, clinical characteristics (age, gender, comorbidities, previous stroke) did not have impact on course of cognitive and depressive symptoms. A limitation in our study was that the number of patients was relatively small, therefore we intend to perform further study with larger case volume to estimate the impact of carotid artery endarterectomy on cognitive functions and depressive symptoms.
\end{abstract}

UDC Classification: 616.8 DOI: http://dx.doi.org/10.12955/cbup.v4.820

Keywords: carotid endarterectomy, cognitive impairment, depressive symptoms.

\section{Introduction}

Traditionally, vascular cognitive impairment was understood entirely as a condition resulting from a symptomatic ischemic or hemorrhagic event, i.e. stroke. However, it is now well recognized that progressive decline in cognitive functioning may result from cerebrovascular disease, even in the absence of a clinically detectable precipitating event (Jokinen et al., 2009). In 2006, the National Institute of Neurological Disorders and the Stroke-Canadian Stroke Network recommended the Montreal Cognitive Assessment (MoCA) test, or some of its subtests, as an optimal brief protocol for the assessment of vascular cognitive impairment (Hachinski et al., 2006, Popovic, Lovrencic-Huzjan, \& Demarin, 2009; Popovic et al., 2011). The MoCA compares equally or favorably to the mini-mental state examination (MMSE) in terms of sensitivity to cognitive impairment and sensitivity to change, over time (Koski, 2013).

Depression is one of the most prevalent and treatable mental disorders presenting in general medical as well as specialty settings (Kroenke \& Spitzer, 2002). Few epidemiologic studies have examined the association between depressive symptoms and atherosclerosis in subjects aged $\geq 65$ years (Faramawi

\footnotetext{
${ }^{1}$ Elina Pucite, Pauls Stradins Clinical University Hospital, Department of Neurology and

Riga Stradins University, Department of Neurology and Neurosurgery, Riga, Latvia, elina.pucite@ @rsu.lv, elina.pucite@gmail.com

${ }^{2}$ Marius Slisers, University of Latvia, Faculty of Medicine, Riga, Latvia

${ }^{3}$ Evija Miglane, Pauls Stradins Clinical University Hospital, Department of Neurology and

Riga Stradins University, Department of Neurology and Neurosurgery, Riga, Latvia

${ }^{4}$ Dainis Krievins, Pauls Stradins Clinical University Hospital, Vascular Surgery Centre and University of Latvia, Faculty of

Medicine, Department of Surgery, Riga, Latvia

${ }^{5}$ Renars Erts, Riga Stradins University, Department of Physics, Riga, Latvia

${ }^{6}$ Kristaps Jurjans, Pauls Stradins Clinical University Hospital, Department of Neurology, Riga, Latvia

${ }^{7}$ Ildze Krievina, Pauls Stradins Clinical University Hospital, Department of Neurology, Riga, Latvia
} 
et al., 2007). In particular, vascular disease has been postulated to increase the risk of later depression (Alexopoulos et al., 1997). The patient health questionnaire(PHQ-9) depression scale is half the length of many other depression measures, has comparable sensitivity, and involves the same nine criteria on which the diagnosis of DSM-IV depressive disorders is based (Kroenke, Spitzer, \& Williams, 2001). The PHQ-9 is a dual-purpose instrument that can establish a provisional depressive disorder diagnosis as well as grade the severity of depressive symptoms (Kroenke \& Spitzer, 2002).

The objective of our study is to evaluate the impact of carotid endarterectomy (CEA) on the cognitive performance of elderly patients with severe symptomatic and asymptomatic carotid disease and clinically important depressive symptoms. This includes exploring the possible associations between certain demographics, clinical characteristics of patients with carotid artery disease, and cognitive function and depression.

\section{Materials and methods}

The prospective single-center study included 48 patients with severe carotid artery stenosis who were referred for endarterectomy to the Paul's Stradins Clinical University hospital. Severe carotid artery stenosis $\geq 70 \%$ was defined according to the North American Symptomatic Carotid Artery Endarterectomy Trial (NASCET) criteria (Barnett et al., 1998). Carotid artery stenosis was estimated using computer tomography angiography. Patients were considered symptomatic if a minor stroke (modified Rankin Scale 0 - III at the time of inclusion) or TIA (transient ischemic attack) had occurred within the previous six months.

Patients under 18 years of age, with progressive cerebral disease (tumor or multiple sclerosis) and major stroke (modified Rankin Scale IV-V), patients with recent ( $<6$ months) antidepressive therapy, and patients not available for long term follow-up were excluded. The study protocol was approved by the corresponding local ethic committee of Riga Stradins University. Written informed consent was obtained from all patients prior to their study inclusion.

Data of patients' gender, age, comorbidities (other atherosclerotic disease, arterial hypertension, chronic heart failure, diabetes mellitus, and atrial fibrillation), lifestyle characteristics (smoking and body mass index) were recorded on a standardized form. Patients were defined as smokers if they were either current or former smokers. Body mass index (BMI) was calculated as weight $(\mathrm{kg})$ divided by the square of height in meters $\left(\mathrm{m}^{2}\right)$.

Neurological examination was performed using the National Institutes of Health Stroke Scale (NIHSS) on all patients before surgery, and then 24 hours and six months after surgery. Functional outcome was assessed using the Barthel Index, which measures the activity of daily living (Aminoff, 2009).

An assessment of cognitive and depressive symptoms was performed by a trained neurologistat 1-3 days before and six months after surgery. Cognitive function was assessed using the MoCA, Latvian and Russian version. The MoCA is 10-minute cognitive screening tool used to detect mild cognitive impairment. The MoCA scores range from 0 to 30 and are divided into seven subscores: visuospatial executive ( $0-5$ points), naming ( $0-3$ points), attention ( $0-6$ points), language ( $0-3$ points), abstraction ( $0-2$ points), memory ( $0-5$ points), and orientation ( $0-6$ points). An additional point is given to each patient who has an educational experience of 12 years or less. A final total score of 26 and above is considered normal (Nasreddine et al., 2005; Nasreddine, 2015).

Depressive symptoms were assessed using the PHQ-9 depression scale, Latvian and Russian version (Vrublevska et al., 2015). A PHQ-9 score can range from 0 to 27, with each of the nine items scoring between 0 ("not at all") and 3 ("nearly every day"). Cut-off points 5, 10, 15, and 20 represent the threshold for mild, moderate, moderately severe, and severe depression. It is recommended to use a PHQ-9 score of 10 or greater as a screening cut-off point, based on sensitivity for major depression of $88 \%$, which corresponds to a specificity of $88 \%$ (Kroenke \& Spitzer, 2002).

\section{Statistical analysis}

Patient numbers and percentages were given as discrete data. Continuous data were presented using means and standard deviations. The changes of the PHQ-9 and MoCA scores were evaluated with a paired sample t test. The Chi-Square test was used to compare groups of categorical data. All tests 
were considered statistically significant at $\mathrm{p}<0.05$. The Cohen's $d$ value was used to estimate the standardized difference between two means. Calculations were performed using the statistical package for social sciences (SPSS) for Windows, version 22.0.

\section{Results}

Characteristics of patients

Our study involved 48 patients with severe carotid artery stenosis $(\geq 70 \%)$ and who had been transferred to Paul's Stradins Clinical University Hospital for endarterectomy. The demographic, lifestyle, and clinical characteristics of the patients are listed in Table 1.

Table 1: Characteristics of patients with severe carotid artery stenosis (total number of patients: 48; mean age: $68 \pm 8$ years; age range: 44 to 81 years)

\begin{tabular}{|c|c|}
\hline Characteristic & Patient number (\% of total) \\
\hline Sex & 1. \\
\hline Male & $33(68.75 \%)$ \\
\hline Female & $15(31.25 \%)$ \\
\hline Life style characteristics: & 2. \\
\hline Smoking & $29(60.42 \%)$ \\
\hline \multicolumn{2}{|l|}{ Body mass index: } \\
\hline$<30 \mathrm{~kg} / \mathrm{m}^{2}$ & $35(72.90 \%)$ \\
\hline$>30 \mathrm{~kg} / \mathrm{m}^{2}$ & $13(27.10 \%)$ \\
\hline \multicolumn{2}{|l|}{ Comorbidity: } \\
\hline Ischemic heart disease & $18(37.50 \%)$ \\
\hline Chronic heart failure & $11(22.92 \%)$ \\
\hline Arterial hypertension & $42(87.50 \%)$ \\
\hline Peripheral artery disease & $12(25.00 \%)$ \\
\hline Diabetes mellitus & $3(06.25 \%)$ \\
\hline Atrial fibrillation & $3(06.25 \%)$ \\
\hline Carotid disease: & 3. \\
\hline Symptomatic & $25(52.08 \%)$ \\
\hline Transient ischemic attack & $10(20.83 \%)$ \\
\hline Minor stroke & $15(31.25 \%)$ \\
\hline Asymptomatic & $23(47.92 \%)$ \\
\hline \multicolumn{2}{|l|}{ Neurological symptoms: } \\
\hline NIHSS 0 & $31(64.60 \%)$ \\
\hline NIHSS $1-5$ & $16(33.30 \%)$ \\
\hline NIHSS 6-10 & $1(02.10 \%)$ \\
\hline \multicolumn{2}{|l|}{ Barthel index } \\
\hline 20 & $37(77.10 \%)$ \\
\hline 19 & $1(02.10 \%)$ \\
\hline 18 & $7(14.60 \%)$ \\
\hline 17 & $1(02.10 \%)$ \\
\hline 16 & $2(04.20 \%)$ \\
\hline
\end{tabular}

NIHSS: National Institutes of Health Stroke Scale

Source: Authors 
The patients included33 (68.75\%) males and 15 (31.25\%) females. The mean age was $68 \pm 8$ years (age range 44-81 years); median age 70 years; the first quartile of age was 63 years; and the third quartile of age was 74 years. Asymptomatic carotid artery disease was observed in $23(47.92 \%)$ patients, while symptomatic carotid artery disease occurred in 25 , with transient ischemic attack relating to $10(20.83 \%)$ and minor stroke to $15(31.25 \%)$.

Most of the patients were free of severe neurological symptoms (16 or 33.30\%) and only one patient had a NIHSS rating below 10, with a result of nine. More than half of the patients had a Barthel Index of 20, and only two patients had a Barthel Index of 16.

More than a third of patients, i.e. 18 (37.50\%), had ischemic heart disease. Peripheral artery disease occurred in $12(25.00 \%)$ patients and diabetes mellitus and atrial fibrillation in three $(6.25 \%)$. As many as $60.42 \%$ were smokers at one time, and $27.10 \%$ were obese. The total MoCA and PHQ-9 scores are given in Table 2.

Table 2: Total MoCA and PHQ-9 scores before and after surgery

\begin{tabular}{|c|r|r|r|r|r|r|}
\hline $\begin{array}{c}\text { Total } \\
\text { MoCA } \\
\text { scores* }\end{array}$ & $\begin{array}{c}\text { Before } \text { surgery } \\
\mathrm{n}(\% \text { of } \mathrm{n})\end{array}$ & $\begin{array}{c}\text { After } \\
\text { surgery } \\
\mathrm{n}(\% \text { of } \mathrm{n})\end{array}$ & & \multirow{4}{*}{$\begin{array}{c}\text { PHQ-9 } \\
\text { scores }\end{array}$} & $\begin{array}{r}\text { Before } \text { surgery } \\
\mathrm{n}(\% \text { of } \mathrm{n})\end{array}$ & $\begin{array}{r}\text { Aftersurgery } \\
\mathrm{n}(\% \text { of } \mathrm{n})\end{array}$ \\
\hline$\geq 26$ & $16(33.3)$ & $24(50.0)$ & & $0-4$ & $22(45.8)$ & $21(43.8)$ \\
\hline $20-25$ & $25(52.1)$ & $20(41.7)$ & & $5-9$ & $15(31.2)$ & $22(45.8)$ \\
\hline$\leq 19$ & $7(14.6)$ & $4(8.3)$ & & $>10$ & $11(23.0)$ & $5(10.4)$ \\
\hline 5.
\end{tabular}

\section{Cognitive performance before and at six months after carotid artery endarterectomy}

The paired sample t-test found that the difference in mean MoCA scores between before $(\overline{\mathrm{X}}=23.37$; $\mathrm{SD} \pm 3.27)$ and after $(\overline{\mathrm{X}}=24.69 ; \mathrm{SD} \pm 3.68)$ surgery was $1.32(95 \% \mathrm{CI}=0.48-2.16)$. This difference was statistically significant ( $p=0.003$; Cohen's $d$ value $=0.95$ ). A more detailed MoCA subscore analysis (Table 3) showed a statistically significant improvement in MoCA memory subscores ( $p=$ 0.003; Cohen's $d$ value $=0.42$ ).

With regard to the risk factors (chronic ischemic heart disease, peripheral artery disease, arterial hypertension, chronic heart failure, atrial fibrillation, or smoking), MoCA subscores were similar for before and after surgery, except in patients with diabetes mellitus. Patients with diabetes mellitus had statistically significant $(p=0.03)$ mean total MoCA scores after the endarterectomy, compared to baseline. Symptomatic or asymptomatic severe carotid artery stenosis had no statistically significant ( $p=0.57$ ) effect on MoCA scores, either before or after CEA.

\section{Depressive symptoms before and at six months after carotid artery endarterectomy}

According to the recommended threshold (PHQ-9 score >10) for screening depressive symptoms, 11 (23\%) patients, with high-grade internal carotid artery stenosis before surgery, had moderate depressive symptoms, while 15 (31.2\%) patients had mild depressive symptoms (Table 2).

The mean PHQ-9 score in the range 5-9 at 6 months after CEA did not decrease statistically significantly from the PHQ-9 scores at baseline $(6.64 \pm 1.6$ vs $6.85 \pm 4.1 ; p=0.8)$. Whereas the paired sample t-test showed a mean PHQ-9 score $>10$ for patients at six months after surgery, CEA (7.5 4 4.6) had decreased significantly ( $p=0.019$; Cohen's $d$ value $=1.32)$ compared with the PHQ-9 scores at baseline $(12.6 \pm 2.8)$. 
By using repeated measurement analysis of variance, we found gender, age, comorbidities, and symptomatic and asymptomatic carotid artery stenosis had no statistical significance $(p<0.05)$ on the course of the PHQ-9 score from baseline to follow-up stage.

Table 3: MoCA scores at baseline and 6 months after operation

\begin{tabular}{|l|r|r|r|}
\hline \multirow{2}{*}{ MoCA } & Subscores before surgery & Subscores after surgery & \multirow{2}{*}{ p-value } \\
\cline { 2 - 3 } & Mean (SD) & Mean (SD) & \\
\hline Visuospatial executive & $3.63(1.13)$ & $3.77(1.19)$ & 0.490 \\
\hline Naming & $2.95(0.48)$ & $2.91(0.48)$ & 0.160 \\
\hline Attention & $5.07(1.20)$ & $5.09(1.23)$ & 0.900 \\
\hline Language & $1.77(0.81)$ & $1.71(0.80)$ & 0.720 \\
\hline Abstraction & $1.42(0.70)$ & $1.56(0.70)$ & 0.260 \\
\hline Memory & $2.18(2.01)$ & $3.00(1.84)$ & $0.003^{*}$ \\
\hline Orientation & $5.81(0.66)$ & $6.07(0.46)$ & 0.040 \\
\hline \multicolumn{4}{|l|}{ 6. } \\
\hline $\begin{array}{l}\text { MoCA: Montreal Cognitive Assessment } \\
\text { SD: standard deviation } \\
* \text { significant result }\end{array}$ \\
\hline Source: Authors & & \\
\hline
\end{tabular}

\section{Discussion}

The presence of carotid artery stenosis has been recognized as a risk factor for stroke and cognitive decline in patients older than 65 years of age (Hachinski, 2007). The CEA is an effective means of preventing future stroke (Halliday et al., 2004), but its contribution to delaying cognitive decline or the onset and prognosis of dementia remains uncertain (Baracchini et al., 2012). Many studies have focused on the neurocognitive course of patients undergoing CAE with controversial results, but few have been performed on the elderly, which is the population at greater risk of cognitive decline and dementia (Baracchini et al., 2012)

In our study, although MoCA scores were adjusted to educational level, the mean total MoCA scores before surgery were mainly in the range of 20 - 25 for mild cognitive impairment (Nasreddine et al., 2005). Our MoCA scores at baseline were lower than in a similar study (Baracchini et al., 2012). A total MoCA score less than 20 before surgery was found in seven (14.6\%) patients, compared to four $(8.3 \%)$ patients after surgery. There was no difference in mean total MoCA scores in patients with either symptomatic or asymptomatic carotid artery stenosis, and so the patients were not subdivided for further analysis. A comparison of mean total MoCA scores showed a statistically significant improvement in cognitive function after CEA, especially in memory subscores. Although different neuropsychological testing protocols were used, a similar pattern of cognitive impairment in patients with severe carotid artery stenosis was found in previous studies (Popovic et al., 2009; Everts et al., 2014; Kim et al., 2015; Baracchini et al., 2012; Kougias et al., 2015).

Depression is commonly observed, not just in patients with ischemic heart disease (Stewart et al., 2003), but also in patients with carotid artery stenosis. Depressive symptoms are common among elderly patients and may lead to severe lifestyle limitations (Mlekusch et al., 2006). Depressive disorders may be causatively associated with degenerative processes based on cerebrovascular disease, a concept that is referred to as "vascular depression". The vascular depression hypothesis suggests that cerebrovascular disease may predispose patients to depressive symptoms (Alexopoulos et al., 1997; Mlekusch et al., 2006). Currently, limited data exists regarding the course of depressive symptoms after successful carotid endarterectomy (Crawley et al., 2000) or after carotid artery stent placement (Mlekusch et al, 2006). Studies have revealed a $12-15 \%$ prevalence of depressive symptoms in patients older than 50 years (Stek et al., 2004). In our study, a total PHQ-9 score $>10$ points involved $11(23 \%)$ patients, none being aware of their emotional status nor ever receiving anti- 
depressive therapy in a primary care setting. The mean PHQ-9 score of patients with high grade internal carotid artery stenosis did not change at six months after CEA $(5.97 \pm 4.40$ vs $5.62 \pm 3.90)$. However, the decrease in frequency of PHQ-9 score $>10$ at six months after CEA was statistically significant compared to the PHQ-9 score at baseline. Although our results differed from a previous study that compared depressive symptoms before and after either CEA or carotid percutaneous transluminal angioplasty (PTA; Crawley et al., 2000), there are several case reports where major depression improved after CEA (Gressier et al., 2011). Similar findings where depressive symptoms improved in patients with severe carotid artery stenosis after PTA are also available, though based on different questionnaires (Mlekusch et al., 2006; Huang et al., 2012).

Our study had limitations in that the number of patients was relatively small and there was no age and sex-matched control group to compare MoCA and PHQ-9 scores. We aim to further this study by assessing the effect of endovascular treatment on cognitive function and depressive symptoms in patients with severe carotid artery stenosis.

\section{Conclusion}

Carotid artery endarterectomy seems to have beneficial effects on the course of cognitive impairment and depressive symptoms in patients with severe carotid artery stenosis. Demographic, clinical characteristics (age, gender, comorbidities, previous stroke) did not have impact on course of cognitive and depressive symptoms. A limitation in our study was that the number of patients was relatively small, therefore we intend to perform further study with larger case volume to estimate the impact of carotid artery endarterectomy on cognitive functions and depressive symptoms.

\section{References}

Alexopoulos, G. S., Meyers, B. S., Young, R. C., Campbell, S., Silbersweig, D., \& Charlston, M. (1997). "Vascular depression" hypothesis. Archives of General Psychiatry, 54, 915-922.

Aminoff (2009). Handbook of Clinical Neurology, Volume 94. Stroke Part III: Investigation and management. Amsterdam, Elsevier. 971 - 1327.

Baracchini, C., Mazzalai, F., Gruppo, M., Lorenzetti, R., Ermani, M., \& Ballotta, E. (2012). Carotid endarterectomy protects elderly patients from cognitive decline: a prospective study. Surgery, 151, 99-106.

Barnett, H. J. M., Taylor, D. W., Eliasziw, M., Fox, A. J., Ferguson, G. G., Haynes, R. B., Rankin, R. N., Clagett, G. P., Hachinski, V. C., Sackett, D. L., Math, K. E. T. M., \& Meldrum. H. E (1998). Benefit of carotid endarterectomy in patients with symptomatic moderate or severe stenosis. New England Journal of Medicine, 339,1415-1425.

Crawley, F., Stygall, J., Lunn, S., Harrison, M., Brown, M. M., \& Newman, S. (2000). Comparison of microembolism detected by transcranial Doppler and neuropsychological sequelae of carotid surgery and percutaneous transluminal angioplasty. Stroke, 31, 1329-1334.

Everts, R., Wapp, M., Burren, Y., Kellner-Weldon F., El-Koussy, M., Jann, K., Lenior, J. D., Michel, P., \& Schroth, G. (2014). Cognitive and emotional effects of carotid stenosis. Swiss Medical Weekly, 144, w13970.

Faramawi, M. F., Gustat, J., Wildman, R. P., Rice, J., Johnson, E., \& Sherwin, R. (2007). Relation between depressive symptoms and common carotid artery atherosclerosis in American persons $\geq 65$ years of age. The American Journal of Cardiology, 1610-1613.

Gressier, F., Khlif, H., Denier, C., Hardy, P., Ducreux, D., \& Corruble, E. (2011). Drug-resistant major depression associated with carotid artery stenosis. American Journal oF Psychiatry, 168, 4, 439.

Hachinski, V., Iadecola, C., Petersen, R. C., Breteler, M. M., Nyenhuis, D. L., Black, S. E., Powers, W. J., DeCarli, C., Merino, J. G., Kalaria, R. N., Vinters, H. V., Holtzman, D. M., Rosenberg, G. A., Wallin, A., Dichgans, M., Marler, J. R., \& Leblanc, G. G. (2006). National Institute of Neurological Disorders and Stroke-Canadian Stroke Network vascular cognitive impairment harmonization standards. Stroke, 37, 2220- 2241.

Hachinski, V. (2007). Stroke and vascular cognition impairment. A transdisciplinary, translational and transactional approach. Stroke, 38, 1396-1403.

Halliday, A., Mansfield, A., Marro, J., Peto, C., Peto, R., Potter, J., \& Thomas, D. (2004). Prevention of disabling and fatal strokes by successful carotid endarterectomy in patients without recent neurological symptoms: randomized controlled trial. Lancet 2004;363:1491-502.

Huang, H., Chen, K., Guo, T., Zhang, Y., Qu, W., Zhou, Z., Liu, G., \& Chen, L. (2012). Treatment with carotid angioplasty stent placement for post-stroke depression compared to antidepressants. Neurosciences, 17(1), 53-56.

Jokinen, H., Kalska, H., Ylikoski, R., Madureira, S., Verdelho, A., van der Flier, W. M., Scheltens, P., Barkhof, F., Visser, M. C., Fazekas, F., Schmidt, R., O’Brien, J., Waldemar, G., Wallin, A., Chabriat, H., Pantoni, L., Inzitari, D., \& Erkinjuntti, T. (2009). Longitudinal cognitive decline in subcortical ischemic vascular disease - the LADIS study. Cerebrovascular Diseases, 27, 384-391. 
Kim, J. J., Schwartz, S., Wen, J., DeVirgilio, C., Lobue, A., Walot, I., Koopmann, M., Donayre, C., \& White, R. A. (2015). Comparison of neurocognitive outcomes after carotid endarterectomy and carotid artery stenting. The American Surgeon, 81,1010-1014.

Koski, L. (2013). Validity and applications of the Montreal cognitive assessment for the assessment $\mathrm{f}$ vascular cognitive impairment. Cerebrovascular diseases,36, 6-18.

Kougias, P., Collins, R., Pastorek, N., Sharath, S., Barshes, N. R., McCulloch, K., Pisimisis, G., \& Berger, D. H. (2015). Comparison of domain specific cognitive function after carotid endarterectomy and stenting. Journal of Vascular Surgery, 62(2), 355-61.

Kroenke, K., Spitzer, R. L., \& Williams, J. B. W. (2001). The PHQ-9: validity of a brief depression severity measure. Journal of general Internal Medicine, 16, 606-613.

Kroenke, K., \& Spitzer, R. L. (2002). The PHQ-9: a new depression diagnostic and severity measure. Psychiatric annals, 3239.

Mlekusch, W., Mlekusch, I., Minar, E., Haumer, M., Kopp, C. W., Ahmadi, R., Lehrner, J., \& Schillinger, M. (2006). Is there improvement of "Vascular depression" after carotid artery stent placement. Radiology, 2, 508-514.

Nasreddine, Z. S. (2015, May). MoCa - Montreal Cognitive Assessment. Retrieved March 12, 2015, from www.mocatest.org.

Nasreddine, Z. S., Phillips, N. A., Bedirian, V., Charbonneau, S., Whitehead, V., Collin, I., Cummings, J. L., \& Chertkow, H. (2005). The Montreal Cognitive Assessment, MoCA: A Brief Screening Tool For Mild Cognitive Impairment. Journal of the American Geriatrics Society, 53(4), 695-699.

Popovic, I. M., Lovrencic-Huzjan, A., \& Demarin, V. (2009). Assessment of subtle cognitive impairment in stroke-free patients with carotid disease. ActaClinicaCroatica, 48, 231-240.

Popovic, I. M., Lovrencic-Huzjan, A., Simundic, A. M., Popovic, A., Seric, V., \& Demarin, V (2011). Cognitive performance in asymptomatic patients with advanced carotid disease. Cognitive and Behavioral Neurology, 24, 145-151.

Stek, M. L., Gussekloo, J., Beekman, A.T., van Tilburg, W., \& Westendorp, R. G. (2004). Prevalence, correlates and recognition of depression in the oldest old: the Leiden 85-plus study. Journal of Affective Disorders, 78, 193-200.

Stewart, R. A., North, F. M., West, T. M., Sharples, K. L., Simes, R. J., Colquhoun, D. M., White, H. D., \& Tonkin, A. M. (2003). Depression and cardiovascular morbidity and mortality: cause or consequence? European Heart Journal, 24, $2027-$ 2037.

Vrublevska, J., Trapencieris, M., Snikere, S., Ivanovs, R., Berzina-Novikova, N., Zikusa, A., \& Rancans, E. (2015). PHQ-9 validation in treatment seeking population in primary care setting in Latvia - the results of the pilot study of the National Research Project. European Neuropsychopharmacology, 25, S365. 